\title{
Iterative Methods for Solving General Nonlinear Quasi-Variational Inequalities
}

\author{
Eman Al-Shemas* \\ Department of Mathematics, College of Basic Education, PAAET, Science and Technology Campus, Al-Ardiya, Kuwait
}

Received: 14 Jan. 2013, Revised: 20 May. 2013, Accepted: 21 May. 2013

Published online: 1 Jan. 2014

\begin{abstract}
This paper presents some iterative methods for solving the general nonlinear quasi-variational inequality problem and consider the convergence criteria of the iterative schemes under certain conditions. Some special cases are also discussed.
\end{abstract}

Keywords: Variational inequalities, quasi- variational inequalities, fixed point methods, projection methods, Wiener-Hopf equations.

\section{INTRODUCTION}

Variational inequalities which were introduced by stampacchia in the early sixties have a considerable impact on a wide class of problems in mechanics, optimization problems, operations research and engineering sciences.

If the convex set does depend upon the solution, then a problem in this class of variational inequalities is called a quasi variational inequality. These were introduced and studied by Benousan and Lions [1]. See ([1]-[20]) and the references therein for numerical methods and applications of variational and quasi-variational inequalities.

In this paper, we study a class of nonlinear quasi-variational inequalities, called the general nonlinear quasi-variational inequalities, and analyze some new iterative algorithms for solving it using the projection technique and the implicit Wiener-Hopf equations technique. Then we discuss the convergence criteria of the iterative algorithms under certain conditions. As several variational classes of variational inequalities and related optimization problems are part of general quasi-variational inequalities, then it is an immediate consequence that the results of this paper hold for these problems.

\section{PRELIMINARIES AND FORMULATION}

Let $\mathrm{H}$ be a real Hilbert space whose inner product and norm are denoted by $\langle.,$.$\rangle and \|$.$\| respectively. Let \mathrm{K}$ be a nonempty closed convex subset of $H$, and $m: K \rightarrow K, T: H \longrightarrow H, A: H \longrightarrow H$ are nonlinear operators. Let $g: H \longrightarrow H$ be a continuous mapping, then we consider the problem of finding $u \in H$ such that $g(u) \in K$, and

$\langle T u, g(v)-g(u)\rangle \geq\langle A(u), g(v)-g(u)\rangle, \forall v \in H: g(v) \in K(u)$,

where $K(u)=m(u)+K$, which we call the general nonlinear quasi-variational inequalities problem denoted by (GNQVIP).

\section{SPECIAL CASES}

I.(a) If $g(v)=v, v \in H$, then problem (1) is equivalent to finding $u \in H$ such that $g(u) \in K(u)$, and

$$
\langle T u, v-g(u)\rangle \geq\langle A(u), v-g(u)\rangle, \forall v \in K(u),
$$

which is known as a general strongly nonlinear quasivariational inequality problem(GSNQVIP) introduced and studied by Siddiqi and Ansari [2].

(b) If $g=I$, the identity mapping, then problem (1) is reduced to finding $u \in K(u)$ such that

$$
\langle T u, v-u\rangle \geq\langle A(u), v-u\rangle, \forall v \in K(u),
$$

which is known as a strongly nonlinear quasi variational inequality problem (SNQVIP), studied by Siddiqi and Ansari [3].

\footnotetext{
*Corresponding author e-mail: emanalshemas@gmail.com
} 
(c) If $m=0$, that is $K(u)=K$, then (1) is called a nonlinear variational inequality, considered by Noor [4].

(d) If $m=0$, that is $K(u)=K$, and $K=H$, then problem (1) reduces to finding $u \in H$ such that

$g(v) \in H$,

$$
\langle T u-A(u), g(v)\rangle=0, \text { for all }
$$

which is known as the weak formulation of the odd order boundary value problems, see Ciarlet et. al. [5].

II. If $A=0$, then

(a) Inequality (1) is equivalent to finding $u \in K(u)$, and

$$
\langle T u, g(v)-g(u)\rangle \geq 0, \forall v \in H: g(v) \in K(u),
$$

Recently Noor et. al.[6] discussed (4) as a special case - Also (4) can be used to study odd order and nonsymmetric abstacle, unilateral and equilibrium problems and other problems of pure and applied sciences. The third-order implicit obstacle boundary value problem of finding $u$ such that

$$
\begin{aligned}
& -u^{\prime \prime \prime} \geq f(x) \quad \text { on } \quad \Omega=[0,1](5) \\
& u \geq M(x, u) \quad \text { on } \quad \Omega=[0,1] \\
& {\left[-u^{\prime \prime \prime}-f(x)\right][u-M(x, u)]=0 \text { on } \quad \Omega=[0,1]} \\
& u(0)=0, \quad u^{\prime}(0)=0, \quad u^{\prime}(1)=0
\end{aligned}
$$

where $f(x)$ is a continuous function and $M(x, u(x))$ is the obstacle function, is considered and studied by Noor et. al.in the framework of variational inequality approach in [6].

(b) If $m=0$, inequality (1) takes the form

$$
\langle T u, g(v)-g(u)\rangle \geq 0, \forall v \in H: g(v) \in K,
$$

which is called a general variational inequality problem, introduced and studied by Noor [7].

(c) If $g(v)=v$, inequality (1) is equivalent to finding $u \in K(u)$, and

$$
\langle T u, v-g(u)\rangle \geq 0, \forall v \in K(u),
$$

which is known as a general quasi variational inequality, introduced by Noor [8].

(d) If $m=0$, and $g(v)=v$, inequality (1) takes the form

$$
\langle T u, v-g(u)\rangle \geq 0, \forall v \in K,
$$

which is called a general variational inequality problem, introduced and studied by Noor [9] and Isac [10].

III. If $A=0, m=0, g=I$, then (1) takes the form

$$
\langle T u, v-u\rangle \geq 0, \forall v \in K,
$$

Problem (9) is called a variational inequality, which was studied by Hartman and Stampacchia [11], see also Lions and Stampacchia [12] and Stampacchia [13].
It is obvious that many classes of variational inequalities can be considered as special cases of the general quasi variational inequality (1).

We need the following concepts and well-known results:

Lemma 2.1. Let $K(u)$ be a closed convex set in $H$. Then, for a given $z \in H, u \in K(u)$ satisfies the inequality

$$
\langle u-z, v-u\rangle \geq 0, \forall v \in K(u)
$$

if and only if $u=P_{K(u)} z$, where $P_{K(u)}$ is the projection of $H$ onto the closed convex set $K(u)$ in $H$.

Lemma 2.2. The mapping $P_{K}$ is nonexpansive, that is,

$$
\left\|P_{K}(u)-P_{K}(v)\right\| \leq\|u-v\|, \forall u, v \in H .
$$

Lemma 2.3. If $K(u)=m(u)+K$ and $K \subset H$ is a closed convex subset, then for any $u, v \in H$, one has

$$
P_{K(u)}(v)=m(u)+P_{K}(v-m(u))
$$

Definition 2.1. An operator $T: H \rightarrow H$ is said to be

i) strongly monotone, if there exists a constant $\alpha>0$ such that

$$
\langle T u-T v, u-v\rangle \geq \alpha\|u-v\|^{2}, \forall u, v \in H ;
$$

ii) Lipschitz continuous if there exists a constant $\beta>0$ such that

$$
\|T u-T v\| \leq \beta\|u-v\|, \forall u, v \in H
$$

The implicit projection operator $P_{K(u)}$ is not nonexpansive. We shall assume that the implicit projection operator $P_{K(u)}$ satisfies the Lipschitz type continuity, which plays an important role in the existence theory and in developing numerical methods for solving (GNQVIP) (1) [6]. Assume that the implicit projection operator $P_{K(u)}$ satisfies the condition

$$
\left\|P_{K(u)} w-P_{K(v)} w\right\| \leq v\|u-v\|, \forall u, v, w \in H
$$

where $v>0$ is a positive constant.

Problem (1) is equivalent to finding $u \in H: g(u) \in K(u) \in$ such that

$$
0 \in T u-A(u)+N_{K(u)}^{P}(g(u)),
$$

where $N_{K(u)}^{P}(g(u))$ denotes the normal cone of $K(u)$ at $g(u)$. Problem (11) is called the quasi-variational inclusion problem associated with quasi-variational inequality (1). This implies that the variational inequality (1) is equivalent to finding a zero of the sum of two monotone operators (11). This equivalent formulation is the core of this paper since it uses the projection operator technique for solving the general nonlinear quasi variational inequality (1). 


\section{ITERATIVE METHODS}

In this section, we establish the equivalence between the variational inequality (GNQVIP) (1) and the fixed point problem using the projection operator technique.

Lemma 3.1. $u \in H: g(u) \in K(u)$ is a solution of the general quasi variational inequality (1) if and only if $u \in$ $H: g(u) \in K(u)$ satisfies the relation

$$
g(u)=P_{K(u)}[g(u)-\rho T u+\rho A(u)],
$$

where $P_{K(u)}$ is the projection operator onto $K(u)$ and $\rho>0$ is a constant.

Proof. One can do the proof by invoking Lemma2.1.

From Lemma 3.1, one can infer that the general nonlinear variational inequality (1) is equivalent to the fixed point problem (12), and consequently formulation (12) can be used to obtain an approximate solution of (1) by an iterative algorithm. The fixed point problem (12) is used to suggest the following iterative methods for solving the (GNQVIP) (1).

\subsection{Projection Iterative Method}

Relation (12) can be written as follows

$$
u=u+\alpha_{n}\left\{-g(u)+P_{K(u)}[g(u)-\rho T u+\rho A(u)]\right\},
$$

where $\alpha_{n} \in[0,1], \forall n \geq 0$.

Equation (13) is used to suggest the following Algorithm

Algorithm 3.1. For a given $u_{0} \in H$, find the approximate solution $u_{n+1}$ by the iterative scheme

$$
\begin{aligned}
& u_{n+1}=u_{n}+\alpha_{n}\left\{-g\left(u_{n}\right)+P_{K\left(u_{n}\right)}\left[g\left(u_{n}\right)-\rho T u_{n}+\rho A\left(u_{n}\right)\right]\right\} \\
& \text { where } \alpha_{n} \in[0,1], \forall n \geq 0 .
\end{aligned}
$$

\subsection{Wiener-Hopf Equations Technique}

Here we consider the problem of solving the general implicit Wiener-Hopf equations. To be more precise, let $P_{K(u)}$ be the projection of $H$ onto the closed convex set $K(u)$ and $Q_{K(u)}=I-P_{K(u)}$, where $I$ is the identity operator. For given nonlinear operators $T, A, g$, consider the problem of finding $z \in H$ such that

$$
T g^{-1} P_{K(u)} z+\rho^{-1} Q_{K(u)} z=A\left(g^{-1} P_{K(u)} z\right),
$$

where we have used the fact that $g^{-1}$ exists. Equation (15) is called the general implicit Wiener-Hopf equation. If $g=I$ and $K(u) \equiv K$, then one can obtain the original Wiener-Hopf equations, which are mainly due to Shi [14].

Now we use Lemma 3.1 to establish the equivalence between problems (1) and (15) which is the main motivation behind the next result.

Lemma 3.2. The general implicit Wiener-Hopf equation (15) has a solution $z \in H$, if and only if, the general nonlinear quasi-variational inequality problem $(G N Q V I P)(1)$ has a solution $u \in K(u)$, provided

$$
\begin{gathered}
u=g^{-1} P_{K(u)} z \\
z=g(u)-\rho(T u-A(u)),
\end{gathered}
$$

where $P_{K(u)}$ is the projection of $H$ onto the closed convex set $K(u)$.

Proof. Let $u \in H: g(u) \in K(u)$ be a solution of (1). Then, form Lemma 3.1, one obtains

$$
u=g^{-1} P_{K(u)}[g(u)-\rho(T u-A(u))]
$$

Let

$$
z=g(u)-\rho(T u-A(u)),
$$

Then, one has

$$
u=g^{-1} P_{K(u)} z
$$

and

$$
z=P_{K(u)} z-\rho T g^{-1} P_{K(u)} z+\rho A\left(g^{-1} P_{K(u)} z\right),
$$

that is

$$
\rho^{-1} Q_{K(u)} z+T g^{-1} P_{K(u)} z=A\left(g^{-1} P_{K(u)} z\right) .
$$

This shows that $z \in H$ is a solution of (15) and the converse is also true. $\square$

Algorithm 3.2. For a given $z_{0} \in H$, find the approximate solution $z_{n+1}$ by the iterative scheme

$$
g\left(u_{n}\right)=P_{K\left(u_{n}\right)} z_{n}, n=0,1,2, \ldots
$$

$$
z_{n+1}=\left(1-\alpha_{n}\right) z_{n}+\alpha_{n}\left[g\left(u_{n}\right)-\rho T u_{n}+\rho A\left(u_{n}\right)\right], n=0,1,2, \ldots
$$

where $0 \leq \alpha_{n} \leq 1$, for all $n \geq 0$. 


\section{CONVERGENCE THEOREMS}

In this section we shall discuss the convergence criteria of the Algorithms 3.1 and 3.2.

The following Lemma will be needed in the proof of Theorem 4.1.

Lemma 4.1. [15] Let $\eta$ and $\sigma$ be positive scalars with $\eta \leq \sigma$. Then for all $\alpha \in[0,1]$,

$$
1-2 \eta \alpha+\sigma^{2} \alpha^{2} \leq\left(1-\alpha+\alpha \sqrt{1-2 \eta+\sigma^{2}}\right)^{2} .
$$

Theorem 4.1. Let the operators $T, g: H \rightarrow H$ be strongly monotone with constants $\alpha>0, \eta>0$ respectively, and Lipschitz continuous with constants with $\beta>0, \sigma>0$ respectively. Suppose that the operators $A, m$ are Lipschitz continuous with constants $\gamma>0, v>0$ respectively. If (10) holds and there exists a constant $\rho>0$ such that

$$
t(\rho)<1-2 v-2 \sqrt{1-2 \eta+\sigma^{2}}
$$

where

$$
t(\rho)=\sqrt{1-2 \alpha \rho+\beta^{2} \rho^{2}}+\gamma \rho
$$

and $\alpha_{n} \in[0,1], \forall n \geq 0, \quad \sum_{n=0}^{\infty} \alpha_{n}=\infty$, then the approximate solution $u_{n}$ obtained from Algorithm 3.1 converges to a solution $u \in H: g(u) \in K(u)$ satisfying the general nonlinear quasi-variational inequality problem (1).

Proof. Let $u \in H: g(u) \in K(u)$ be a solution of (GNQVIP)(1). Then, From (13)and (14), we have

$$
\begin{aligned}
&\left\|u_{n+1}-u\right\|=\| u_{n}-u-\alpha_{n}\left(g\left(u_{n}\right)-g(u)\right) \\
&+ \alpha_{n}\left\{P_{K\left(u_{n}\right)}\left[g\left(u_{n}\right)-\rho T u_{n}+\rho A\left(u_{n}\right)\right]\right. \\
&\left.-P_{K(u)}[g(u)-\rho T u+\rho A(u)]\right\} \| \\
& \leq\left\|u_{n}-u-\alpha_{n}\left(g\left(u_{n}\right)-g(u)\right)\right\| \\
& \leq \quad \alpha_{n} \| P_{K\left(u_{n}\right)}\left[g\left(u_{n}\right)-\rho T u_{n}+\rho A\left(u_{n}\right)\right] \\
&-P_{K(u)}[g(u)-\rho T u+\rho A(u)] \| \\
&\left\|u_{n}-u-\alpha_{n}\left(g\left(u_{n}\right)-g(u)\right)\right\| \\
&+\alpha_{n} \| P_{K\left(u_{n}\right)}\left[g\left(u_{n}\right)-\rho T u_{n}+\rho A\left(u_{n}\right)\right] \\
&-P_{K(u)}\left[g\left(u_{n}\right)-\rho T u_{n}+\rho A\left(u_{n}\right) \|\right. \\
&+\alpha_{n} \| P_{K(u)}\left[g\left(u_{n}\right)-\rho T u_{n}+\rho A\left(u_{n}\right)\right. \\
&-P_{K(u)}[g(u)-\rho T u+\rho A(u) \|
\end{aligned}
$$

$$
\begin{aligned}
\leq & \left\|u_{n}-u-\alpha_{n}\left(g\left(u_{n}\right)-g(u)\right)\right\| \\
& +2 \alpha_{n} v\left\|u_{n}-u\right\| \\
& +\alpha_{n}\left\|u_{n}-u-\left(g\left(u_{n}\right)-g(u)\right)\right\| \\
& +\alpha_{n}\left\|u_{n}-u-\rho\left(T u_{n}-T u\right)\right\| \\
& +\rho\left\|A\left(u_{n}\right)-A(u)\right\|
\end{aligned}
$$

from Lemma 2.3 and the Lipschitz continuity of $m$, one has

$$
\begin{aligned}
\left\|P_{K\left(u_{n}\right)} w-P_{K(u)} w\right\| & =\| m\left(u_{n}\right)-m(u)+P_{K}\left(w-m\left(u_{n}\right)\right) \\
& -P_{K}(w-m(u)) \| \\
& \leq 2\left\|m\left(u_{n}\right)-m(u)\right\| \\
& \leq 2 v\left\|u_{n}-u\right\|, \\
& \forall u, u_{n} \in H,
\end{aligned}
$$

where $w=g\left(u_{n}\right)-\rho T u_{n}+\rho A\left(u_{n}\right)$.

from the strong monotonicity and Lipschitz continuity of $g$, we have

$$
\begin{aligned}
& \left\|u_{n}-u-\alpha_{n}\left(g\left(u_{n}\right)-g(u)\right)\right\|^{2} \leq \\
& \left\|u_{n}-u\right\|^{2}-2 \alpha_{n}\left\langle g\left(u_{n}\right)-g(u), u_{n}-u\right\rangle+\alpha_{n}^{2}\left\|g\left(u_{n}\right)-g(u)\right\|^{2}
\end{aligned}
$$

$$
\leq\left(1-2 \alpha_{n} \eta+\alpha_{n}^{2} \sigma^{2}\right)\left\|u_{n}-u\right\|^{2}
$$

and

$$
\begin{aligned}
\left\|u_{n}-u-\left(g\left(u_{n}\right)-g(u)\right)\right\| & \leq \\
\left\|u_{n}-u\right\|^{2}-\left\langle g\left(u_{n}\right)-g(u),\right. & \left.u_{n}-u\right\rangle+\left\|g\left(u_{n}\right)-g(u)\right\|^{2} \\
& \leq\left(1-2 \eta+\sigma^{2}\right)\left\|u_{n}-u\right\|^{2} .
\end{aligned}
$$

from Lemma 4.1 we have

$$
\begin{gathered}
\left\|u_{n}-u-\alpha_{n}\left(g\left(u_{n}\right)-g(u)\right)\right\| \leq \\
\left(1-\alpha_{n}+\alpha_{n} \sqrt{1-2 \eta+\sigma^{2}}\right) \\
\left\|u_{n}-u\right\| .
\end{gathered}
$$

Similarly from the strong monotonicity and Lipschitz continuity of $T$, One obtains

$$
\begin{aligned}
\left\|u_{n}-u-\rho\left(T\left(u_{n}\right)-T(u)\right)\right\| & \leq\left\|u_{n}-u\right\|^{2}- \\
& \rho\left\langle T\left(u_{n}\right)-T(u), u_{n}-u\right\rangle \\
& +\rho^{2}\left\|T\left(u_{n}\right)-T(u)\right\|^{2} \\
\leq & \left(1-2 \alpha \rho+\beta^{2} \rho^{2}\right)\left\|u_{n}-u\right\|^{2}
\end{aligned}
$$


from (19),(21), (22), (23), and using the Lipschitz continuity of $A$, we have

$$
\begin{aligned}
\left\|u_{n+1}-u\right\| \leq & \left\{1-\alpha_{n}+\alpha_{n} \sqrt{1-2 \eta+\sigma^{2}}\right. \\
& +2 \alpha_{n} v+\alpha_{n} \sqrt{1-2 \eta-\sigma^{2}} \\
& +\alpha_{n} \sqrt{1-2 \alpha \rho+\beta^{2} \rho^{2}} \\
& \left.+\alpha_{n} \gamma \rho\right\}\left\|u_{n}-u\right\| \\
\leq & \left\{1-\alpha_{n}\left(1-2 \sqrt{1-2 \eta+\sigma^{2}}-2 v\right.\right. \\
& \left.-\sqrt{1-2 \alpha \rho+\beta^{2} \rho^{2}}-\gamma \rho\right\}\left\|u_{n}-u\right\| \\
\leq & \left\{1-\alpha_{n}\left(1-2 v-2 \sqrt{1-2 \eta+\sigma^{2}}-t(\rho)\right)\right\} \\
& \left\|u_{n}-u\right\| \\
= & \left(1-\alpha_{n} \theta\right)\left\|u_{n}-u\right\| \\
\leq & \prod_{i=0}^{n}\left(1-\alpha_{i} \theta\right)\left\|u_{0}-u\right\|
\end{aligned}
$$

where $\theta=1-2 v-2 \sqrt{1-2 \eta+\sigma^{2}}-t(\rho)$

$$
\text { and } t(\rho)=\sqrt{1-2 \alpha \rho+\beta^{2} \rho^{2}}+\gamma \rho
$$

From (18), it follows that $\theta>0$, and since $\sum_{n=0}^{\infty} \alpha_{n}=$ $\infty$ we have $\lim _{n \rightarrow \infty}\left\{\prod_{i=0}^{n}\left(1-\alpha_{i} \theta\right)\right\}=0$, Consequently the sequence $\left\{u_{n}\right\}$ converges strongly to $u \in H: g(u) \in K(u)$. This completes the proof. $\square$

Now we shall prove the convergence of the iterative scheme of Algorithm 3.2.

Theorem 4.2. Let $T, g$ be strongly monotone with constant $\alpha>0, \eta>0$ respectively, and Lipschitz continuous with constant $\beta>0, \sigma>0$ respectively. Let the operators A, $m$ are Lipschitz continuous with constant $\gamma>0, v>0$ respectively. If (10) holds and there exists a constant $\rho$ such that (18) holds and $\alpha_{n} \in[0,1], \forall n \geq 0$; $\sum_{n=0}^{\infty} \alpha_{n}=\infty$, then the approximate solution $z_{n}$ obtained from Algorithm 3.2 converges to a solution $z \in H$ satisfying the general implicit Wiener-Hopf equation (15).

Proof. Let $z \in H$ be a solution of (15). Then, using Lemma 4.2, we get

$$
z=\left(1-\alpha_{n}\right) z+\alpha_{n}\{g(u)-\rho(T u-A(u))\},
$$

where $0 \leq \alpha_{n} \leq 1$.

from (17) and (24),one has

$$
\begin{aligned}
\left\|z_{n+1}-z\right\| \leq & \left(1-\alpha_{n}\right)\left\|z_{n}-z\right\| \\
& +\alpha_{n}\left\|u_{n}-u-\left(g\left(u_{n}\right)-g(u)\right)\right\| \\
& +\alpha_{n}\left\|u_{n}-u-\rho\left(T u_{n}-T u\right)\right\| \\
& +\alpha_{n} \rho\left\|A\left(u_{n}\right)-A(u)\right\|
\end{aligned}
$$

Since the operators $T, g$ are strongly monotone with constant $\alpha>0, \eta>0$ and Lipschitz continuous with constant $\beta>0, \sigma>0$, and using the Lipschitz continuity of the operator $A$ with constant $\gamma>0$, it follows from (21), (23) that

$$
\begin{aligned}
\left\|z_{n+1}-z\right\| & \leq\left(1-\alpha_{n}\right)\left\|z_{n}-z\right\| \\
& +\alpha_{n}\left(\sqrt{1-2 \eta+\sigma^{2}}+\sqrt{1-2 \rho \alpha+\rho^{2} \beta^{2}}+\rho \gamma\right) \\
& \left\|u_{n}-u\right\|
\end{aligned}
$$

from (10), (20), (21) and the fact that $g(u)=P_{K(u)} z$, we have

$$
\begin{aligned}
\left\|u_{n}-u\right\| & =\left\|u_{n}-u-\left(g\left(u_{n}\right)-g(u)\right)+\left(P_{K\left(u_{n}\right)} z_{n}-P_{K(u)} z\right)\right\| \\
& \leq\left\|u_{n}-u-\left(g\left(u_{n}\right)-g(u)\right)\right\|+\left\|\left(P_{K\left(u_{n}\right)} z_{n}-P_{K\left(u_{n}\right)} z\right)\right\| \\
& +\left\|\left(P_{K\left(u_{n}\right)} z-P_{K(u)} z\right)\right\| \\
& \leq \sqrt{1-2 \eta+\sigma^{2}}\left\|u_{n}-u\right\|+\left\|z_{n}-z\right\|+2 v\left\|u_{n}-u\right\|
\end{aligned}
$$

$$
\begin{aligned}
\left\|u_{n}-u\right\| & \leq \frac{1}{\left(1-2 v-\sqrt{1-2 \eta+\sigma^{2}}\right)}\left\|z_{n}-z\right\| \\
& \leq \frac{1}{k}\left\|z_{n}-z\right\|
\end{aligned}
$$

where $k=1-2 v-\sqrt{1-2 \eta+\sigma^{2}}$,

Now (25) can be written as

$$
\begin{aligned}
\left\|z_{n+1}-z\right\| & \leq\left(1-\alpha_{n}\right)\left\|z_{n}-z\right\| \\
+ & \frac{\alpha_{n}}{k}\left(1-2 v-k+\sqrt{1-2 \rho \alpha+\rho^{2} \beta^{2}}+\rho \gamma\right)\left\|z_{n}-z\right\| \\
& \leq\left(1-\alpha_{n}\right)\left\|z_{n}-z\right\|+\alpha_{n} \phi\left\|z_{n}-z\right\|
\end{aligned}
$$

where

$$
\phi=\frac{1}{k}\left(1-2 v-k+\sqrt{1-2 \rho \alpha+\rho^{2} \beta^{2}}+\rho \gamma\right)
$$

From (18), we see that $\phi<1$ and consequently

$$
\begin{aligned}
\left\|z_{n+1}-z\right\| & \leq\left[\left(1-(1-\phi) \alpha_{n}\right]\left\|z_{n}-z\right\|\right. \\
& \leq \prod_{i=0}^{n}\left[\left(1-(1-\phi) \alpha_{i}\right]\left\|z_{0}-z\right\|\right.
\end{aligned}
$$

Since $\sum_{n=0}^{\infty} \alpha_{n}$ diverges, and $1-\phi>0$, it follows that $\lim _{n \rightarrow \infty} \prod_{i=0}^{n}\left[\left(1-(1-\phi) \alpha_{i}\right]=0\right.$. Consequently the sequence $\left\{z_{n}\right\}$ convergences strongly to $z$ in $H$ satisfying the general implicit Wiener-Hopf equation (15). $\square$ 


\section{References}

[1] A. Benssousan and J. L. Lions, Applications des Inequations Variationelles en Control et en Stochastiques, Dunod, Paris, (1978).

[2] A. H. Siddiqi and Q. H. Ansari, General Strongly Nonlinear Variational inequalities, J. Math. Anal. Appl., 166, 386-392 (1992).

[3] A. H. Siddiqi and Q.H. Ansari, Strongly Nonlinear Quasivariational Inequalities, J. Math. Anal. Appl., 149, 444-450 (1990).

[4] M. A. Noor, On Nonlinear Variational Inequalities, Internat. J. Math. \& Matt. Sea., 14, 399-402 (1991).

[5] P. G. Clarlet, M. H.Schultz, and R. S.Varga, Numerical Methods of High Order Accuracy for Nonlinear Boundary Value Problems, V. Monotone Operator Theory, Numer. Math., 13, 51-77 (1969).

[6] K. Tahata, K. Yamamoto and S. Tomizawa, Decomposition of Symmetry Using Palindromic Symmetry Model in a Two-Way Classification, Journal of Statistics Applications \& Probability, 1, 201-204 (2012).

[7] M. A. Noor, Strongly Nonlinear Variational Inequalities, C. R. Math. Rep. Acad. Sci.Canada, 4, 213-218 (1982).

[8] M. A. Noor, Quasi-variational Inequalities, Appl. Math. Lett. I, 367-370 (1988).

[9] M. A. Noor, General Variational Inequalities, Appl. Math. Lett. 1, 119-122 (1988).

[10] G. Isac, A Special Variational Inequality and The Implicit Complementarity Problem, J. Fac. Sci. Univ. Tokyo, 37, 109-127 (1990).

[11] P. Hartman and G. Stampacchia, On Some Nonlinear Elliptic Differential Functional Equations, Acta Math., 115, 153-188 (1966).

[12] J. L. Lions and G. Stampacchia, Variational Inequalities, Comm. Pure Appl. Math., 20, 493-512 (1967).

[13] G. Stampacchia, Formes Bilineaires Coercitives Sur Les Ensembles Convexes, C. R. Acad. Sci, Paris, 258, 44134416 (1964).

[14] P. Shi, Equivalence of Variational Inequalities with WienerHopf Equations, Proc. Amer. Math. Soc., 111, 339-346 (1991).

[15] E. Al-Shemas and S. Billups, An Iterative Method for Generalized Set-Valued Nonlinear Mixed Quasi-variational Inequalities,J. Comp. Appl.Math., 170, 423-432 (2004).

[16] E. Al-Shemas, Resolvent Operator Method for General Variational Inclusions, J. Math. Inequal., 3, 455-462 (2009).

[17] E. Al-Shemas, Projection Iterative Methods for Multivalued General Variational Inequalities, Appl. Math. \& Inform. Sc., 3, 177-184 (2009).

[18] A. S. Antipin, N. Mijajlovic, M. Jacimovic, A Second-order Iterative Method for Solving Quasi-variational inequalities, Comp. Math. Math. Physics, 53, 258-264 (2013).

[19] C. Baiocchi and A. Capelo, Variational and Quasivariational Inequalities, J. Wiley and Sons, New York, (1984).

[20] D. Kinderlehrer and G. Stampacchia, An Introduction to Variational Inequalities and Their Applications, SIAM, Philadelphia, (2000).

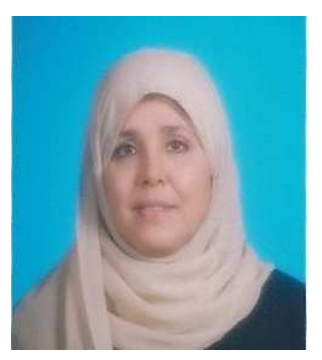

Eman H. N. Al-Shemas received her $\mathrm{Ph} . \mathrm{D}$. in 1991. She now works as an Associate Professor in Mathematics Department, College of Basic Education, Paaet, Kuwait and has been serving as the Head of the Department since 2007. She published several papers in various international refereed journals and eight Arabic text books. 